\title{
Chartis-Phänotypen prognostizieren Erfolg
}

Hintergrund und Fragestellung: Die endoskopische Lungenvolumenreduktion (ELVR) wird seit mehreren Jahren bei Patienten mit schwerer COPD und Lungenemphysem durchgeführt. Zur Evaluation der kollateralen Ventilation kann das ChartisSystem (Chartis, Pulmonx, USA) verwendet werden. Patienten, die CV-negativ (kein Hinweis auf Kollateralventilation) sind, haben eine deutlich bessere Erfolgsquote als jene Patienten, die $\mathrm{CV}$-positiv sind.

Zur Messung wird bronchoskopisch ein Ballonkatheter in den $\mathrm{zu}$ verschließenden Lappen eingeführt, der Lappen völlig blockiert und der Fluss aus dem Lappen gemessen. Fällt der Fluss ab und steigt der Druck im Lungenlappen, ist davon auszugehen, dass keine CV vorliegt. Das Signal auf dem Monitor ist aber nicht immer eindeutig und die Autoren dieser Studie möchten eine Definition der Chartis-Flußtypen darstellen und diese mit dem Outcome nach Ventilimplantation vergleichen.

Patienten u. Methoden: Retrospektiv wurden ChartisMessungen von 166 Patienten analysiert. 52 waren CV-negativ und erhielten Ventile zur Volumenreduktion. Auch Originalie

Herzog D, Thomsen C, Poellinger $A$ et al. Outcomes of Endobronchial Valve Treatment Based on the Precise Criteria of an Endobronchial Catheter for Detection of Collateral Ventilation under Spontaneous Breathing. Respiration 2016;91(1):69-78.
- CV-positiv: kein Flussabfall und kein Anstieg der Widerstandes (Rrt).

- CV-negativ: Abfall des Flusssignals mit entsprechendem Anstieg von Rrt.

- Low flow: Plötzlicher Abfall des Flusses und Anstieg des Widerstandes.

- Low Plateau, LP, Abfall des Flusssignals, bis zu einem Plateau ohne weiteren Abfall, Rrt steigt nicht.

Ergebnisse: Die Interpretation der Chartis-Kurven ist durch mehrere Betrachter gut reproduzierbar. Alle CV-positiv-Messsungen zeigten nach 4 Minuten immer noch einen Fluss von über 50\% des Ausgangsflusses. Alle CV-negativ-Messungen hatten einen Abfall des Flusssignals auf $<50 \%$ des Ausgangsflusses und $<20 \%$ nach 5 Minuten. Der LP-Phänotyp zeigte ebenfalls einen Flussabfall auf $<50 \%$ mit Plateau bei $>20 \%$. LF zeigte eine raschen Abfall des Flusses auf null innerhalb von 30s.

Bezüglich des Widerstandes blieben CV-positiv und LP $<3$ $\mathrm{cmH}_{2} \mathrm{O}$ x s/ml in $5 \mathrm{~min}$. Alle CV-Messungen zeigten einen Widerstand von $>3 \mathrm{~cm} \mathrm{H}_{2} \mathrm{O}$. Die LF-Messungen zeigten einen Rn$\mathrm{dx}$-Anstieg auf über $100 \mathrm{~cm} \mathrm{H}_{2} \mathrm{O}$ x s/ml innerhalb der ersten 30s.

Die 52 Patienten, die Ventile erhielten, waren CV-negativ in den Oberlappen (21), CV-negativ in den Unterlappen (13) und 12 zeigten ein LF-Phänomen. 18 bzw. 9 dieser Patienten zeigten eine signifikante TLVR (target lobe volume reduction), Verbesserung des $\mathrm{FEV}_{1}$ und VC.

Schlussfolgerung: Die exakte Definition der Phänotypen vereinfacht die Differenzierung bzgl. der Kollateralventilation und liefert ein Flussdiagramm als Orientierung vor Ventilimplantation.

\section{- Kommentar von Dr. med. Maren Schuhmann}

\section{Chartis besonders für Zentren hilfreich, die mit der ELVR beginnen}

Die Chartis-Messung wird regelmäßig bei Patienten angewandt, um CV vor Ventilimplantation auszuschließen. Hierbei kann es Probleme mit der Abdichtung oder mit Sekret sowie Husten geben, und das System ist daher nicht immer zuverlässig. Gelingt der Verschluss, kommt es zu unterschiedlichen Kurven, die im Idealfall eindeutig positiv oder negativ sind. Es gibt aber auch Kurven, die sich nicht eindeutig sind. Bisher waren nur eindeutig CVnegative Patienten beschrieben, die von ELVR mittels Ventilen profitieren. In dieser Studie nun werden genaue Cut-off-Kriterien bestimmt, um die Kurven den Phänotypen zuzuordnen. Besonders beim LF-Phänotyp ist schwierig zu erklären, wie er zustande kommt: ob durch Kontakt des Katheters mit der Bronchialschleimhaut oder durch peripheren Bronchialkollaps. Die Autoren geben zwar an, dass diese Patienten von Ventilen profitieren, allerdings handelt es sich hier nur um eine sehr kleine Stichprobe $(n=12)$. Die Untersuchungen erfolgeten in Lokalanästhesie. Oft werden sie aber in Vollnarkose gemacht, und es gilt zu sehen, ob die Ergebnisse auf eine mechanische Ventilation übertragbar sind.
Eine Definition der Phänotypen ist aber hilfreich, besonders zur Orientierung für Zentren, die erst mit der ELVR beginnen.

$\mathrm{Zu}$ beachten ist auch, dass die Chartis-Ergebnisse immer auch mit der CT bewertet werden müssen, um das individuell beste Vorgehen festlegen zu können. Idealerweise sollten die Patienten in Zentren behandelt werden, in denen Expertise für Chartis-Messung und CT-Interpretation vorhanden ist.

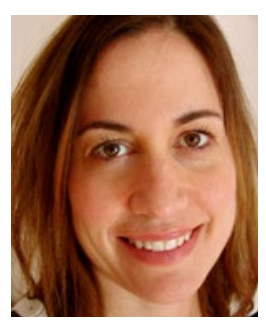

Dr. med. Maren Schuhmann

Thoraxklinik am Universitätsklinikum Heidelberg Röntgenstr. 1, 69126 Heidelberg maren.schuhmann@med.uni-heidelberg.de 